\title{
Correction: Mortality and secondary complications four years after traumatic spinal cord injury in Cape Town, South Africa
}

\author{
(C) International Spinal Cord Society 2020
}

V. Madasa · B. Boggenpoel · J. Phillips · C. Joseph

Correction to: Spinal Cord Series and Cases 6:84 (2020) https://doi.org/10.1038/s41394-020-00334-w, published online 04 September 2020
The original version of this article contained an error in the author affiliations. The institute name [Stellenbosch University] was omitted in affiliation 2 . This has now been corrected in both the PDF and HTML versions of the article. 